\title{
Cardiac-gated Slit Lamp Videography as a Novel Approach to Assessing a Microcirculatory Network
}

\author{
Paul F. Brennan ${ }^{1,2}$, Dewar D. Finlay ${ }^{2}$, Mark S. Spence ${ }^{1}$,Agnes Awauh ${ }^{2}$, James A.D. McLaughlin ${ }^{2}$, \\ Jonathan E. Moore ${ }^{2}$, M. Andrew Nesbit ${ }^{2}$, Emanuele Trucco ${ }^{3}$, Ruixuan Wang ${ }^{3}$, Tara C.B. Moore ${ }^{2}$ \\ ${ }^{1}$ Royal Victoria Hospital, Belfast, United Kingdom \\ ${ }^{2}$ University of Ulster, Belfast, United Kingdom \\ ${ }^{3}$ University of Dundee, Dundee, United Kingdom
}

\begin{abstract}
In this article we describe the development of a system that is designed to image and in turn, allow characterization of the microcirculation in conjunctiva. To characterise the blood flow the system simultaneously captures high quality imagery alongside information on the cardiac cycle, using a multimodal image and biosignal acquisition approach. Images are captured using a smartphone camera attached, via a bespoke adapter, to a 40x magnification slit lamp. Images are recorded as videos at a resolution of up to approximately 1920 x 1080 pixels per frame (i.e. 1080k) and sampled at 60 frames per second. Information on the cardiac cycle is captured using a phonocardiogram device. In order to allow time synchronisation of the video with the phonocardiogram, the phonocardiogram output signal is fed via a custom adapter to the audio input of the smartphone video recording device. This allows for the phonocardiogram to be time synchronised in the audio layer of the video file.

This software system provides the opportunity for cardiac gated assessment of a readily obtainable microvascular network, namely the conjunctival vasculature.
\end{abstract}

\section{Introduction}

The microcirculation is well recognized as the site where the earliest manifestations of CVD, especially inflammatory responses, occur that may play a pivotal role in driving the atherosclerotic process in blood vessels [1]. It is hypothesized that structural and functional changes in the microcirculation easily accessible and clearly visible at the front of the eye, in the conjunctiva, can reflect early inflammation and endothelial dysfunction within the macrovascular circulation such as the coronary and cerebral arteries.

The conjunctival vessels have been previously studied in systemic conditions associated with macrovascular disease, such as hypertension and diabetes mellitus $[2,3]$. The conjunctival vessels are readily accessible for imaging and subsequent physiological analysis. Axial blood flow velocity and vessel diameter provide insight into the microcirculatory physiology.

\section{Previous relevant work}

The microvascular physiology represents that seen in larger vessels. An initial study, using a retinal functional imager (RFI), accurately acquired axial blood flow velocities within the conjunctival vasculature in a range of $0.86-0.99 \mathrm{~mm} / \mathrm{s}$ in five healthy patients [4].

Use of the functional slit-lamp biomicroscope (FSLB) to study the conjunctival physiology has previously shown promise. FSLB was used, in one study, to assess the conjunctival vasculature in six healthy patients. Axial blood flow velocity, vessel diameter, blood flow rate and fractal dimension were all reproducibly measured and quantified [5]. These semi-automated measurements allow for direct analysis of the fundamental physiological properties of the conjunctival vessels.

An automated image analysis method using an FSLB and digital charged coupled device camera was applied in a single study of 15 healthy subjects. Using Frangi filtering [6] on a time-averaged image, vessels were segmented and subsequent measurements were obtained including vessel diameter (D), axial blood velocity (V), wall sheer resistance (WSR) and blood flow detection (Q) [7]. Results, from this study, indicated that there was no significant relationship between D and V in arterioles. There was, however, a statistically significant increase in $\mathrm{V}$ for increased venule diameter. Axial blood velocity was significantly lower in venules compared to arterioles, 
as was WSR. Conjunctival Q increased with larger diameter segments as expected.

Challenges encountered in achieving automated analysis, in the aforementioned studies, include rapid eye movement and blinking which lead to image blurring. One technique to detect such blurred video frames is based on pixel intensity gradient, where blurred frames often have significant lower average gradients over frame pixels than clear frames. Alternatively, the blur issue can be reduced by automatically stabilizing images during video capture based on tracking eye motion and realigning optical path in real time [8]. The use of external fixation targets on the slit FSLB also has been used in these studies to minimize eye movement and allow for continuous acquisition of images throughout the conjunctival regions.

In one study measurement variability, dependent on the time of the day that image acquisition was occurred, was studied in 20 healthy subjects. Each subject was imaged every two hours between $9 \mathrm{am}-5 \mathrm{pm}$. There were no significant differences in vessel $\mathrm{D}$ and haemodynamic parameters during this time frame but they did observe significantly increased fractal dimensions at $3 \mathrm{pm}$ and 5pm compared to $9 \mathrm{am}[9]$.

\section{Current technical developments}

The current acquisition system is based on several separate components that have been integrated to allow capture of ocular imagery in parallel with temporal information relating to the cardiac cycle.

\subsection{Image acquisition}

Image acquisition is achieved via two main hardware components. Firstly, primary illumination and magnification of the ocular vascular structure is achieved using a conventional slit lamp. The currently employed slit lamp is a Topcon SL-D4 [10], and is capable of providing a maximum magnification of 40x. Secondly imagery provided by the slit lamp is further magnified and stored using a smartphone based camera operating in video record mode. The smartphone used in the current system is an Apple iPhone 6s [11]. A number of video record settings have been tested and the current configuration is set to record at a resolution of $1920 \mathrm{x}$ 1080 and at 60 frames per second. The iPhone video recorder is capable of providing a further magnification of $3 x$. Coupling of the smartphone and slit lamp is achieved using a bespoke adapter developed by Zarf Enterprises [12]. This adapter allows attachment of the smartphone to the eyepiece of the slit lamp.

\subsection{Cardiac Cycle Information}

A number of solutions were considered for providing information on the cardiac cycle. These included ECG and Phonocardiogram (PCG) systems. The main challenge was in the identification of a commercial device/system that would output data in real time to allow storage in parallel with the captured video described above. The final choice was made based on the ease of integration with the camera hardware. The chosen solution was PCG acquired using a commercially available "One" electronic stethoscope unit developed by Thinklabs [13]. This unit provides audio output of the PCG via an inbuilt $3.5 \mathrm{~mm}$ headphone jack.

PCG and image acquisition (video) were synchronised by feeding the audio output from the stethoscope into the microphone input on the smartphone video recorder. In order to permit this connection custom adapter circuitry was developed. This circuitry served two purposes. Firstly, it allowed connection of the $3.5 \mathrm{~mm}$ headphone output of the stethoscope to the $3.5 \mathrm{~mm}$ microphone input of the smartphone. This was achieved by providing cross over of the mic/headphone channels and suitable decoupling circuitry to block DC. Secondly, a headphone connection was added to allow an operator to monitor the PCG signal as it is acquired and passed to the smartphone mic input.

\section{Clinical Protocol}

We aim to comprehensively understand the effect of cardiovascular disease on the conjunctival microvasculature and evaluate if the microcirculation can be used as a predictor of Major Adverse Cardiac Events (MACE).

We currently are assessing microvascular physiological parameters that are indicative of endothelial dysfunction, alongside cardiac biomarkers, which could be used as prognostic indicators of MACE.

We are currently studying three population groups. Group one consists of patients deemed at low/no risk for cardiovascular disease. This patient cohort are subsequently being age/sex matched to group two. Group two represent a high-risk group of patients post-ST segment elevation myocardial infarction (STEMI) and hence have established major cardiovascular disease. Group three consists of patients with cyanotic congenital heart disease in whom chronic hypoxaemia leads to endothelial dysfunction and microcirculatory changes.

All patients will have a "Q-RISK" score calculated, a well validated risk assessment tool used to predict the risk of having a myocardial infarction or cerebrovascular accident over the next ten years [14]. This will allow us risk stratify patients within each group studied.

Patients are recruited into the study in adherence with the 
Declaration of Helsinki.

Conjunctival vascular image acquisition, using the protocol previously described, is applied to the temporal and nasal hemispheres in both eyes. We then image the upper and lower quadrant for each ocular hemisphere and thus acquire eight videos per patient studied. Video sampling time is 12 seconds per quadrant, which allows sufficient time for cardiac cycle gating, optimal vessel selection and eradication of images acquired during blinking or eye movement.

An external fixation target is applied to minimize blinking and eye movement.

Once the images are acquired we subsequently then apply the automated analysis methods described previously, including Frangi filtering for vessel segmentation, and also subsequent measurement of vessel diameter, velocity, wall sheer resistance and flow. These microvascular physiological results are analyzed, alongside a cardiovascular demographic risk assessment and cardiac biomarker profile, to establish cardiovascular trends among the three groups studied.

Prior to recruitment, we studied 10 healthy patients to ensure technique was optimized for image acquisition and that, using the software available, quantitative parameters were readily reproducible for analysis. All 10 subjects were studied without complication, while eye movement and blinking were minimized using the techniques described previously.

We aim to compare the physiological microcirculatory parameters, described previously, between our patient groups to better understand the microcirculatory dysfunctional changes expected in the high risk groups. These findings, in turn, may then lead to the development of an adjunctive MACE screening tool using the conjunctival microcirculation in addition to conventional measures such as blood pressure, diabetic status, smoking status etc.

\section{Initial Results}

Using the protocol, described previously, we have been able to successfully achieve vessel segmentation

[Figure 1.].

By applying our automated image analysis method we have generated quantitative measurements, for all segmented vessels, which include vessel width, axial flow velocity, cross-sectional velocity, blood volume flow (Q) and wall sheer resistance.

As we are still in the preliminary stages of the study, it is too early to comment on observed trends between each patient group and the quantitative objectives studied. Upon completion of patient recruitment and data collection we will be able to comment on the relationship, if any, between conjunctival microvascular physiological changes and the groups studied. Upon completion, we will also be able to comment on any trends observed between patient risk and the cardiovascular biomarkers measured in the study.

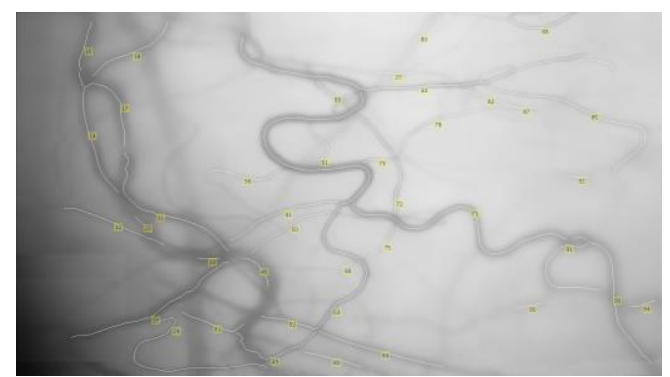

Figure 1. Conjunctival vessel segmentation.

\section{Discussion}

Assessing a patients risk of cardiovascular disease and MACE requires a well validated screening tool, and comprehensive assessment, of individual risk factors in conjunction with relevant biomarker assessment. Using the automated image analysis methods, described previously, the conjunctival microcirculatory haemodynamics can be assessed. We can, therefore, improve upon our own understanding of the microcirculation and if observed haemodynamic abnormalities have any relationship with macrovascular disease.

\section{Acknowledgements}

Funding provided by Northern Ireland Chest Heart and Stroke.

\section{References}

[1] Lockhart CJ, Hamilton PK, Quinn CE, McVeigh GE Endorgan dysfunction and cardiovascular outcomes; The role of the microcirculation. Clin Sci 2009;116(3):175-90

[2] To W et al. Real-time studies of hypertension using nonmydriatic fundus photography and computer-assisted intravital microscopy, Clin. Hemorheol. Microcirc., vol. 53, no. 3, pp. 267-279, 2013.

[3] Cheung A et al. Correlation of microvascular abnormalities and endothelial dysfunction in type-1 diabetes mellitus (T1DM): A realtime intravital microscopy study, Clin. Hemorheol. Microcirc., vol. 42, no. 4, pp. 285-295, 2009.

[4] Wang J. Human conjunctival microvasculature assessed with a retinal functional imager (RFI). Microvascular Res. 2013 Jan; $85: 134-37$ 
[5] Jiang et al. Functional slit lamp biomicroscopy for imaging bulbar conjunctival microvasculature in contact lens wearers. Microvascular Res. 2014 Mar;92:62-71.

[6] Frangi, A. F., Niessen, W. J., Vincken, K. L., \& Viergever, M. A., Multiscale vessel enhancement filtering. Medical Image Computing and Computer-Assisted Interventation, 130-137, 1998.

[7] Khansari, M. M., Wanek, J., Felder, A. E., Camardo, N. \& Shahidi, M. Automated assessment of hemodynamics in the conjunctival microvasculature network. IEEE Transactions on Medical Imaging 35, 605-611, 2016.

[8] Felder, A. E., Cesare, M., Wanek, J., et al., Automated RealTime Conjunctival Microvasculature Image Stabilization. IEEE Transaction on Medical Imaging, 35(7), 1670-1675, 2016.

[9] Xu Z et al Measurement variability of the bulbar conjunctival microvasculature in healthy subjects using functional slit lamp biomicroscopy (FSLB). Microvascular Res. 2015 Sep; 101:15-9

[10] Topcon Medical Systems Inc., USA, www.topconmedical.com

[11] Apple, Inc., USA, www.apple.com

[12] Zara Enterprises USA, http://www.zarfenterprises.com/

[13] Think Medical LLC, USA, http://www.thinklabs.com

[14] Hippisley-Cox J et al. Predicting cardiovascular risk in England and Wales: prospective derivation and validation of QRISK2, BMJ 2008;336:1475-82.

Address for correspondence

Dr Paul Brennan

Cardiology Research Department, West Wing, Royal Victoria Hospital, Belfast, Co.Antrim, BT 12 6BA

paul.brennan@belfasttrust.hscni.net 